\title{
Distributed Resources Coordination inside nearly-Zero Energy Buildings Providing Grid Voltage Support from a Symmetrical Component Perspective
}

\author{
Pablo Arboleya ${ }^{1}$, Pablo Garcia, Bassam Mohamed, Cristina Gonzalez-Moran \\ LEMUR Research Group, Dept. Electrical Engineering, University of Oviedo \\ Torres Quevedo Building, block 4, Gijon Campus, Asturias, Spain
}

\begin{abstract}
In this work, the authors propose a new coordination method for Smart Distribution Networks (SDN) with nearly-Zero Energy Buildings (nZEB). The proposed coordination procedure considers a central controller that runs a state estimation of the grid and calculates the optimum set-points for the buildings. Inside each building, the Building Management System (BMS) manages the converters used by the Dispatchable Generators (DG), the Renewable Generators (RG) and the Energy Storage System (ESS) to fulfil (as far as possible), the central controller request.

In the paper, the unbalanced behaviour of the grid is considered, so the optimum references for the symmetrical component currents in the converters are calculated. The proposed method reduces the network unbalance and the total losses. In addition, buildings with energy surplus can support other neighbours with energy deficit. The present paper is focused on how the BMS calculates the optimum references for all converters placed inside each building. The main contribution of the paper is the use of the symmetrical component approach at building level in order to generate the best references for all converters inside the building.
\end{abstract}

(c) 2016 Published by Elsevier Ltd.

Keywords: Nearly-Zero Energy Buildings, Microgrid Coordination, Distributed Energy Resources, Symmetrical Components.

\begin{tabular}{|c|c|c|c|}
\hline \multicolumn{2}{|c|}{ Nomenclature } & EES & Energy Storage System. \\
\hline \multicolumn{2}{|c|}{ Acronyms } & EMS & Energy Management System. \\
\hline BFS & Backward/Forward Sweep. & nZEB & nearly-Zero Energy Building. \\
\hline BMS & Building Management System. & OPF & Optimal Power Flow. \\
\hline CHP & Combined Heat and Power. & $\mathbf{R G}$ & Renewable Generator. \\
\hline dG & Dispatchable Generator. & RMS & Root Mean Square. \\
\hline DG & Distributed Generation. & SDN & Smart Distribution Network. \\
\hline
\end{tabular}

\footnotetext{
This work was partially supported by the Spanish Ministry of Science and Innovation under Grant ENE2013-44245-R (MICROHOLO Development of a Holistic and Systematical Approach to AC Microgrids Design and Management).

Email address: arboleyapablo@uniovi.es, garciafpablo@uniovi.es, mohamedbassam@uniovi.es, gonzalezmorcristina@uniovi.es (Pablo Arboleya ${ }^{1}$, Pablo Garcia, Bassam Mohamed, Cristina Gonzalez-Moran)

${ }^{1}$ Corresponding author.
} 
VPD

Voltage Power Droop.

Variables

\section{$f$}

$P$

$Q$

I

V

$R$

$X$

Vectors

$\mathbf{P}$

Q

I

V

Subscripts

$D$

$S$

$R$

$L$

$a n, b n, c n$

$a, b, c$

$p, n, z$

Frequency.

Active Power.

Reactive Power.

Current.

Voltage.

Resistance.

Reactance.

Renewable Generator.

Building Load.

Phases a,b and c.
Vector containing all active powers.

Vector containing all reactive powers.

Vector containing all currents.

Vector containing all voltages.

Dispatchable Generator.

Energy Storage Device.

Phases $\mathrm{a}, \mathrm{b}$ and $\mathrm{c}$ to neutral respectively.

Positive, negative and zero sequences respectively. $i$

Subindex for referring to a kind of generator, it can take the values $D, S$ or $R$.

$j \quad$ Subindex for referring to a specific phase, it can take the values $a, b$ or $c$.

Superscripts

min, $\max$ Minimum and maximum respectively applied to active powers and currents.

full, empty Full and empty indicators. applied to the energy storage systems state.

$f \quad$ Four leg indicator applied to the converters state.

av

Relative to the available renewable active power.

opt $1 \quad$ Obtained in the first optimisation stage.

opt 2 Obtained in the second optimisation stage.

opt3 Obtained in the third optimisation stage.

\section{Functions}

PFC Vector function containing all power flow equations.

$T G_{N C S} \quad$ Target function for negative compensation stage (first stage).

$T G_{Z C S}$

$T G_{P C S}$

$T G_{N S S}$
Target function for zero compensation stage (second stage).

Target function for positive compensation stage (third stage).

Target function for network support stage (fourth stage).

\section{Introduction}

Nowadays, the energy management inside smart distribution networks (SDN) is becoming a very important issue. The SDN concept is shown to be the most reliable and effective way to reach high Distributed Generation (DG) penetration levels without putting in risk the network stability and also increasing the power quality. A novel way

5 for embedding DG within SDNs is the so-called nearly-Zero Energy Buildings (nZEB). A nZEB is described as a building in which the net imported energy from the electric grid should be "nearly" zero within an interval of a year [1]. The penetration of these kind of buildings in the European stage will not be negligible since the European Union Directive 2010/31/UE established that all new buildings must be nZEB after 2020.

Regarding the energy management at SDN level, there are basically three philosophies. The first one is based on 10 a central controller that provides references to all devices installed within the network [2]. For instance, in [3, 4], the authors propose a complex coordination system in order to accommodate cooling, heating and power sources within a microgrid. The energy management is then approached like a classical dispatching problem. In [5] the authors propose an Optimal Power Flow (OPF) based tool for short term operation of radial distribution systems under unbalanced conditions. The OPF reduces the total losses as well as the overload and voltage violation risk. In [2], the authors use three solid state switching devices to transfer the single phase loads (houses) from one phase to another. There is a central microgrid controller that calculates real time optimum power flows to obtain the most 
adequated references for all switches in the microgrid. In [6] the authors optimise the cost of a hybrid microgrid based on a central controller that actuates over a Energy Management System (EMS).

The second approach is the distributed management approach. In this case the DGs create their own references depending on local electrical measurements. Considering that most of the DGs are connected to the grid by means of power converters, most of the authors have proposed distributed controls based on droop characteristics [7]. Some authors proposed modifications with respect to the conventional $\mathrm{P}-\mathrm{f} / \mathrm{Q}-\mathrm{V}$ droop. For instance in [8] and [9], the authors proposed the use of a $\mathrm{P}-\mathrm{V}$ droop and a $\mathrm{V}-\mathrm{I}$ droop respectively. In [7], the authors proposed a conventional $\mathrm{P}-\mathrm{f} / \mathrm{Q}-\mathrm{V}$ droop control for a converter based distributed resources. They studied the stability limits of a distribution system with embedded DG. In this case, all the mathematical models were developed in $d q$ synchronous reference frame and they assumed that the system was balanced. One of the special characteristics of the distribution networks (low $\mathrm{X} / \mathrm{R}$ ratio) made the authors propose variations to the conventional droop control. It can be stated that in low distribution networks with low $\mathrm{X} / \mathrm{R}$ ratios is widely accepted among the authors the use of P-V droops also addressed as VPD (Voltage Power Droop). [10, 11, 12, 13].

In [8], the conventional $\mathrm{P}-\mathrm{f} / \mathrm{Q}-\mathrm{V}$ droop is replaced by the $\mathrm{P}-\mathrm{V}$ droop, but they implement a droop for each phase. In [9], the same authors propose a V-I droop control. In [14], the authors consider a special four-leg inverter control for working at distribution level in microgrids. The zero and negative sequence current injection is considered and the authors propose to connect this special converter near the most sensitive loads. In this case, only when the voltage unbalance reaches a given threshold, the compensation is activated and the voltage unbalance is reduced.

Finally, the hybrid approach considers a central controller combined with the distributed controls of the DGs. In [15], the DGs use a droop control, but the droop parameters are calculated by a central controller.

Regarding the nZEB, in most of the cases, a central Building Management System (BMS) calculates the references for the generators and energy storage systems. In [16], the authors consider a building with combined heat and power (CHP) and energy storage systems (ESS) and they propose a day ahead algorithm to schedule generation and consumptions using the classical dispatching problem approach. The authors do not consider neither electrical variables beyond the active powers or interaction with the network. Something similar is proposed in [17]. In this case, a single dwelling is modelled using the single ideal node concept in which all appliances, storage systems and distributed generators are connected. In [18], the BMS decides how to manage the ESS depending on the load and the available generation to reduce the impact of the nZEB with the network. In [19] the buildings demand-side

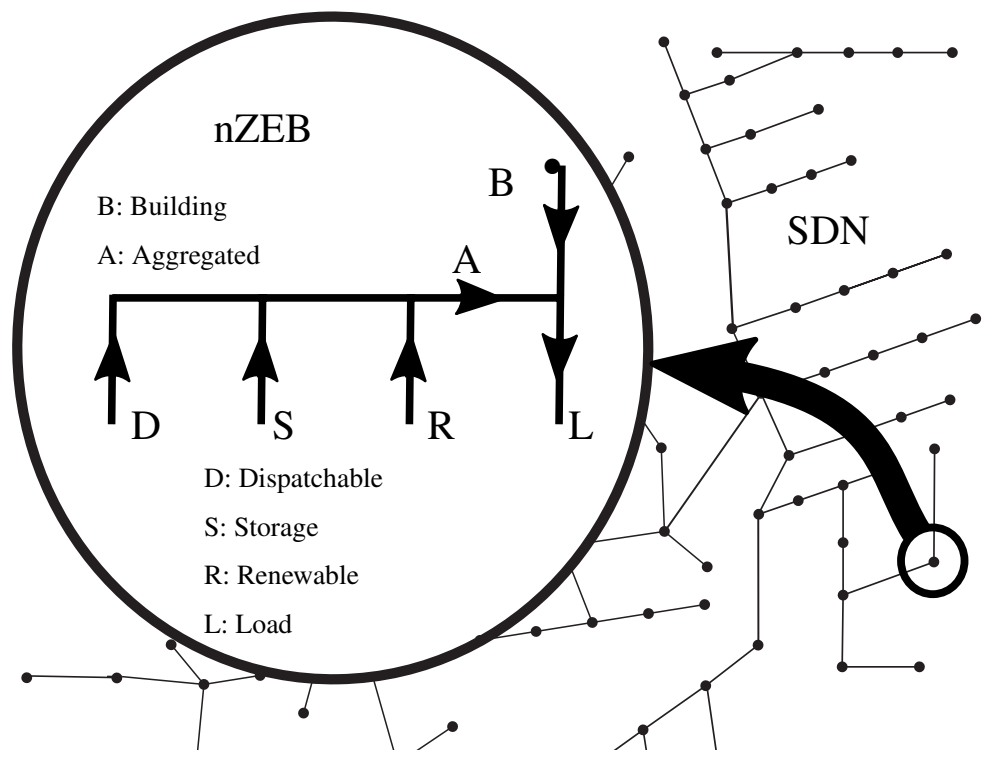

Figure 1. nearly-Zero Energy Building (nZEB) representation embedded in a Smart Distribution Network (SDN). Inside each buildings, different types of generators are considered, dispatchable generators, and renewable generators, as well as loads and Energy Storage systems. 
management is considered as an additional tool for coordinate loads, storage systems and generators inside a microgrid.

In this paper, the authors propose a hybrid strategy applied to smart distribution residential networks, in which dGs and ESSs are installed inside nZEBs. From the point of view of the SDN, each nZEB will be considered as a droop generator if there exists a surplus of energy inside the building. If not, the building will demand the necessary energy from the network. The SDN central controller is in charge of collecting the data sent from all buildings (based in Smart meters and converter measurements), estimating the state of the grid, sending references for tuning the building droops and sharing the state of the network with the BMSs. At building level, each BMS will receive information about the state of the grid and the setup for its droop. Then, the BMS will calculate the references for all the devices inside the building (Dispatchable and Renewable Generators and Energy Storage Systems).

This work is focused on the method to obtain the internal references for the building devices by means of an Interior Point Cascaded Optimisation approach with an embedded Backward/Forward Sweep (BFS) Power Flow. The authors assume that the SDN central controller provide the state of the grid and a specific set point for the buildings droops. Once the internal management is carried out the effect of the buildings over the network is also considered. The unbalanced behaviour of the system is taken into account and all the formulation is expressed using symmetrical components. In [20, 21], a detailed method for estimating the state of a SDN using smart meters data is presented. In

60 [15], an Optimal Power Flow (OPF) approach implemented in the SDN central controller that obtains the best droop values is detailed. The proposed BMS cascaded optimisation will have four nested optimisation stages. In the first, second and third stages, the BMS will try to compensate respectively, the self-consumed negative, zero (in case of inverters with four legs) and positive sequence currents. In the fourth stage the BMS will make the whole building emulate the behaviour of a droop based generator. The building will be able to support the grid if there exists an energy 5 surplus. With this approach, the BMS assigns priority to compensate the self-consumption over the grid support.

The paper is structured as follows; In section II the overall proposed procedure, explaining the inter-actuation between the grid and the buildings is described. In section III, the BMS cascaded optimisation procedure for determining the current references inside the building is detailed. In section IV, two different scenarios are analysed. Section V summarises the laboratory setup and describes the errors obtained when comparing the theoretical implementation with the real measurements. Finally, the conclusions will be presented in section V.

\section{Overall coordination procedure}

In the proposed approach, a SDN with a typical distribution network topology is considered. At each network node (see Fig 11, a building or an aggregation of buildings is connected. In this work, for the sake of simplicity, the authors assume that each node represents a single building that can be conventional or nZEB. The nZEB can be

75 equipped with Dispatchable, non-dispatchable Renewable Generation and Energy Storage Systems. All devices can be connected by means of three-leg or four-leg converters.

With a low sampling rate (15 minutes), determined by the building smart meters setup, all BMSs send a summary report to the SDN central controller with the information of what is happening inside the building. This report should contain at least, the information about the voltages (RMS) at the nZEB point of coupling and the net powers absorbed so or injected from/to the network. All data will be collected asynchronously by the BMS of each building. In this case, the building receives information from other buildings states each 15 minutes too. The packages containing the information from the buildings will be sent to the SDN central controller asynchronously. The central controller run a state estimation algorithm with the asynchronous measures (see [20, 21]). Then, an OPF algorithm is used to calculate the optimal set-points for building droops in order to reduce the total losses. In [15] an example of how this OPF algorithm works is detailed.

Then, the SDN central controller sends the calculated set points to the nZEBs. At building level, each BMS runs the cascaded optimisation procedure with the embedded BFS power flow. The final target is the optimum current reference for each converter in order to fulfil what the central controller requests. However, the cascaded optimisation procedure will assign priority to fulfil the self consumption. It will try to generate first the negative and zero sequence self-consumed currents and then the positive one. Finally, it will adapt the converters set-points to emulate the droop behaviour and fulfil the central controller request, if it is possible.

The present paper is focused on the cascaded optimisation procedure used by the BMS to generate the converter references. 


\section{BMS cascaded sharing procedure}

The BMS will run a cascaded optimisation to generate the current references for the power converters. During the optimisation, all buildings demands in the rest of the network, as well as the droop slope in the studied building are assumed as inputs. These buildinginputs are provided by the SDN central controller at a low sampling rate (15 minutes in our particular case of study). They will be considered as constants during the whole sampling period. The information provided by the building about its own devices will be constantly updated and can be summarised as follows:

- The states of the dispatchable generator, the storage equipment and the renewable generator $\left(s_{D}, s_{S}, s_{R}\right)$. The state will be 0 if the equipment is damaged or not available.

- The voltage at the point of coupling (phase to neutral) $\left(V_{a n}, V_{b n}, V_{c n}\right)$, and the current demanded by the load $\left(I_{L a}, I_{L b}, I_{L c}\right)$.

- The active and reactive powers per phase demanded by the loads inside the building $\left(P_{L a}, P_{L b}, P_{L c}\right)$ and $\left(Q_{L a}, Q_{L b}, Q_{L c}\right)$.

- Fourth-Leg converter activation flag $\left(s_{D}^{f}, s_{S}^{f}, s_{R}^{f}\right)$; Each converter can be configured as four-leg converter, in such case the activation flag will be set to one and the converter would be able to inject zero sequence current.

- The maximum and minimum total active power in each converter (Dispatchable, Storage and Renewable) will be represented as $\left(P_{D}^{\max }, P_{S}^{\max }, P_{R}^{\max }\right)$ and $\left(P_{D}^{\min }, P_{S}^{\min }, P_{R}^{\min }\right)$ respectively.

- The maximum current per phase in each converter $\left(I_{D}^{\max }, I_{S}^{\max }, I_{R}^{\max }\right)$.

- The renewable available active power $\left(P_{R}^{a v}\right)$.

- The battery sate of charge; $s_{S}^{\text {full }}$ will be 1 if the storage device is full, 0 otherwise. $s_{S}^{\text {empty }}$ will be 1 if the storage device is empty, 0 otherwise.

During the whole optimisation process two sets of constraints will be considered. The first one, related with the situation inside the building and labelled as Building Constraints $(\boldsymbol{B C})$ can be described as:

- Active power boundary constraints in the converters; $P_{D}, P_{S}$ and $P_{R}$ are respectively the powers delivered or absorbed by the three converters.

$$
P_{i}^{\min }<P_{i}<P_{i}^{\max } \quad \mid i \in\{D, S, R\}
$$

- Per phase converter current constraints. The subindices $a, b$ and $c$ represent the phase. For instance, $I_{S b}$ represents the current in the phase $b$ of the energy storage device.

$$
\left|I_{i j}\right|<I_{i}^{\max } \quad \mid i \in\{D, S, R\} \wedge j \in\{a, b, c\}
$$

- Zero sequence current injection constraints in the converters with three legs. As it can be observed, the zero sequence currents $\left(I_{D z}, I_{S z}, I_{R z}\right)$ will be set to zero in those converters with three legs.

$$
\left(1-s_{i}^{f}\right) \cdot I_{i z}=0 \quad \mid i \in\{D, S, R\}
$$

- Active power constraint in the renewable generator. The converter reference in the renewable generator must be set in order to inject all the available renewable power.

$$
P_{R}=P_{R}^{a v}
$$


The other set of constraints to be considered is the one labeled as PFC Power Flow Constraints. This set of constraints contain basically the network power flow equations, considering that all network buildings except the studied one are PQ nodes. The values of active and reactive powers in the buildings are the ones estimated by the SDN central controller and will be considered constant during the optimisation process. The next set of equations represents the PFC:

$$
P F C(\mathbf{V}, \mathbf{I}, \mathbf{P}, \mathbf{Q}, k)=0
$$

Where $\mathbf{V}, \mathbf{I}, \mathbf{P}$ and $\mathbf{Q}$ are vectors including the voltages, currents active and reactive powers estimated for all buildings, and $\mathrm{k}$ is the droop assigned to the studied building. In [6] a detailed analysis of all equations contained in the power flow constraints can be found.

During the cascade optimisation procedure different target functions will be considered and specific constraints will be added to the above described sets of constraints. In the first stage of optimisation the BMS will try to compensate the self-consumed negative current. The target function labeled as $T G_{N C S}$ (Target function for negative compensation stage) is the next one:

$$
\min \left(T G_{N C S}\right)=\min \left(I_{D n}+I_{S n}+I_{R n}-I_{L n}\right)
$$

Where $I_{D n}, I_{S n}, I_{R n}$ and $I_{L n}$ represent the negative component currents in the dispatchable generator, the storage system, the renewable generator and the building load. In the second stage, the optimised values of the negative currents obtained from the previous stage $I_{D n}^{o p t 1}, I_{S n}^{o p t 1}$ and $I_{R n}^{o p t 1}$ are incorporated to the building constraints as:

$$
I_{\text {in }}=I_{\text {in }}^{\text {opt } 1} \quad \mid i \in\{D, S, R\}
$$

In this stage, the target function will try to compensate the self-consumed zero sequence current. It will be labeled as $T G_{Z C S}$ (Target function for zero compensation stage) and expressed as follows:

$$
\min \left(T G_{Z C S}\right)=\min \left(I_{D z}+I_{S z}+I_{R z}-I_{L z}\right)
$$

This optimisation stage, will be executed only if any converter is configured as a four-legs converter.If not, the constraints in the expression (7) will be added to the building constraints in the third optimisation stage. The third stage will try to compensate the self consumed positive sequence current. First, the optimised values of the zero sequence current will be added to the building constraints (according to the expression (97)). In case that there is no converter with four-legs configuration, the optimised values will be set automatically to zero.

$$
I_{i z}=I_{i z}^{o p t 2} \quad \mid i \in\{D, S, R\}
$$

The target function in the third stage will be labeled as $T G_{P C S}$ (Target function for positive compensation stage) and will be expressed as:

$$
\min \left(T G_{P C S}\right)=\min \left(I_{D p}+I_{S p}+I_{R p}-I_{L p}\right)
$$

Where $I_{D p}, I_{S p}, I_{R p}$ and $I_{L p}$ represent the positive component currents in the Dispatchable Generator, Storage System, Renewable Generator and Building Load.

The fourth stage of optimisation is launched only if the third one was completely achieved. That means the building is making a full self compensation. In such case the building will try to inject/absorb positive current in phase with the voltage according to the next droop based equation:

$$
\begin{array}{r}
I_{D p}+I_{S p}+I_{R p}=I_{D p}^{o p t 3}+I_{S p}^{o p t 3}+I_{R p}^{o p t 3}+\ldots \\
\ldots-\frac{V_{p}}{\left|V_{p}\right|}\left(I_{0}-k \cdot\left(|V p|-V_{0}\right)\right)
\end{array}
$$

Where $I_{D p}^{o p t 3}, I_{S p}^{o p t 3}$ and $I_{R p}^{o p t 3}$ are the positive currents optimised in the third stage, $V_{p}$ is the positive voltage in the point of coupling of the building with the network (phase to neutral), $V_{0}$ is the modulus of the rated phase-to-neutral voltage 


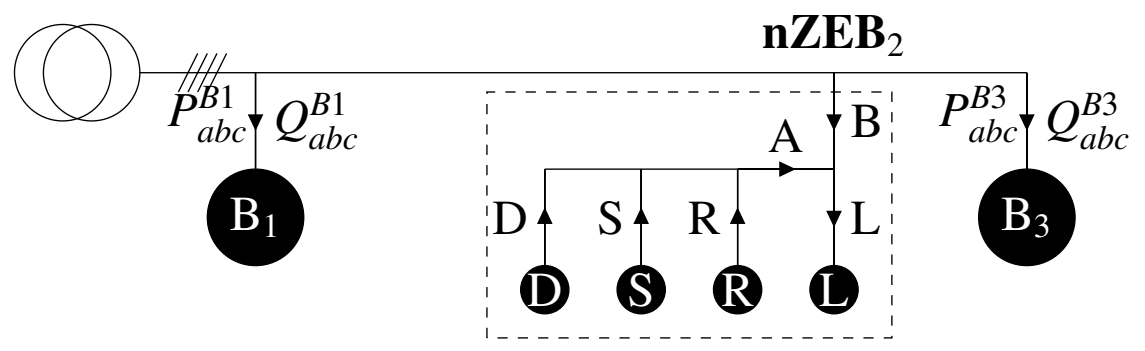

Figure 2. Case of study scheme

and $k$ is the droop slope provided by the SDN central controller. The value of $I_{0}$ is not fixed, it is determined during the optimisation process and it adds a degree of freedom to the droop equation. As it can be observed, the extra positive current injected in the fourth optimisation stage is in phase with the voltage, so the droop is actually a P-V droop. In this stage, the equation (11) will be added to the Power Flow Constraints (PFC) and the target function for this network support stage $T G_{N S S}$ is the next one:

$$
\min \left(T G_{N S S}\right)=\min \left(||\left|V_{p}\right|-V_{0} \|\right)
$$

With this approach, in the fourth stage, the building will support the network, absorbing active power if the voltage is too high or injecting active power if the voltage is too low. The positive current injection will depend on the droop, but always fulfilling the building internal constrains. Another kind of droop for providing also reactive power to the network could be implemented. However, at this voltage level the lines reactive consumption can be neglected and most of the building reactive demand is self-generated. Under these assumptions a proper network voltage support can be achieved with the proposed approach. For solving the cascade optimisation, the authors used an interior point algorithm. When an evaluation of the PFC (Power Flow Constraints) is needed, an embedded Backward/Forward Sweep algorithm is executed.

\begin{tabular}{|c|c|c|c|c|c|c|c|c|c|c|c|c|c|}
\hline \multicolumn{2}{|c|}{$V_{a n}\left(\mathrm{~V},{ }^{\circ}\right)$} & \multicolumn{2}{|c|}{$V_{b n}\left(\mathrm{~V},{ }^{\circ}\right)$} & \multicolumn{2}{|c|}{$V_{c n}\left(\mathrm{~V},{ }^{\circ}\right)$} & \multicolumn{2}{|c|}{$V_{n g}\left(\mathrm{~V},{ }^{\circ}\right)$} & \multicolumn{2}{|c|}{$V_{p}\left(\mathrm{~V},{ }^{\circ}\right)$} & \multicolumn{2}{|c|}{$V_{n}\left(\mathrm{~V},{ }^{\circ}\right)$} & \multicolumn{2}{|c|}{$V_{z}\left(\mathrm{~V},{ }^{\circ}\right)$} \\
\hline Mod. & Ang. & Mod. & Ang. & Mod. & Ang. & Mod. & Ang. & Mod. & Ang. & Mod. & Ang. & Mod. & Ang. \\
\hline 207.2 & 0.5 & 219.4 & -119.9 & 207.8 & 119.9 & 9.9 & 56.3 & 211.5 & 0.2 & 4.6 & 121.9 & 3.3 & -123.7 \\
\hline
\end{tabular}

Table 1. Voltages in $a b c$ reference and in positive, negative and zero sequence at the point of coupling of the nZEB with the grid (Scenario 1).

\begin{tabular}{c|cc|cc|cc|cc} 
& $P_{a}$ & $Q_{a}$ & $P_{b}$ & $Q_{b}$ & $P_{c}$ & $Q_{c}$ & \multicolumn{2}{c}{ Total } \\
& $\mathrm{kW}$ & $\mathrm{kVAr}$ & $\mathrm{kW}$ & $\mathrm{kVAr}$ & $\mathrm{kW}$ & $\mathrm{kVAr}$ & $\mathrm{kW}$ & $\mathrm{kVAr}$ \\
\hline \hline Building & -1.32 & 0.05 & -1.50 & 0.00 & -1.34 & -0.05 & -4.2 & 0.0 \\
Load & 5.00 & 1.64 & 7.00 & 2.30 & 13.00 & 4.20 & 25.0 & 8.1 \\
Aggregat. & 6.32 & 1.59 & 8.50 & 2.30 & 14.34 & 4.25 & 29.2 & 8.1 \\
Dispatch. & 2.27 & 0.60 & 3.00 & 0.76 & 4.73 & 1.41 & 10.0 & 2.8 \\
Storage & 1.79 & 0.40 & 2.52 & 0.77 & 4.86 & 1.42 & 9.2 & 2.6 \\
Renew & 2.26 & 0.06 & 2.99 & 0.76 & 4.74 & 1.42 & 10.0 & 2.8 \\
\hline & \multicolumn{2}{|c|}{$I_{a}\left(\mathrm{~A},{ }^{\circ}\right)$} & \multicolumn{2}{c}{$I_{b}\left(\mathrm{~A},{ }^{\circ}\right)$} & \multicolumn{2}{c}{$I_{c}\left(\mathrm{~A},{ }^{\circ}\right)$} & \multicolumn{2}{c}{$I_{n}\left(\mathrm{~A},{ }^{\circ}\right)$} \\
& Mod. & Ang. & Mod. & Ang. & Mod. & Ang. & Mod. & Ang. \\
\hline \hline Building & 9.2 & -179.8 & 9.2 & 60.2 & 9.2 & -59.8 & 0.0 & 0.0 \\
Load & 36.7 & -20.0 & 45.3 & -138.2 & 94.5 & 104.5 & 53.9 & -64.9 \\
Aggregat. & 45.5 & -16.0 & 54.1 & -135.1 & 103.4 & 105.9 & 53.9 & -64.9 \\
Dispatch. & 16.4 & -16.6 & 19.0 & -134.3 & 34.2 & 105.7 & 16.1 & -64.9 \\
Storage & 12.8 & -14.4 & 16.2 & -137.0 & 35.0 & 106.1 & 21.4 & -64.9 \\
Renew & 16.3 & -16.6 & 19.0 & -134.3 & 34.3 & 105.7 & 16.3 & -64.9
\end{tabular}

Table 2. Powers and peak currents in $a b c$ reference in the whole building. The building net power is positive when it is demanded from the grid. The converter aggregated power is positive when it is delivered to the building (Scenario 1). 


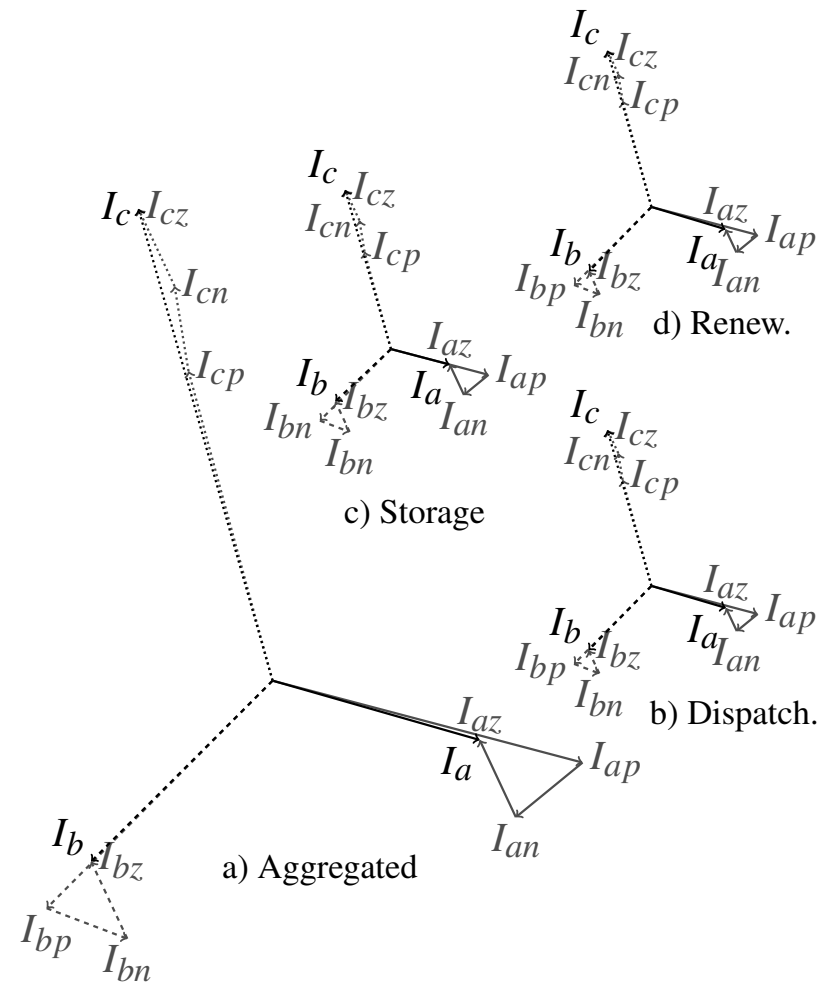

Figure 3. Phasor diagram of the optimised current injection for each converter and also the aggregated phasor diagram considering all converters (Scenario 1).

\begin{tabular}{c|cc|cc|cc} 
& $\begin{array}{c}P_{p} \\
\mathrm{~kW}\end{array}$ & $\begin{array}{c}Q_{p} \\
\mathrm{kVAr}\end{array}$ & $\begin{array}{c}P_{n} \\
\mathrm{~kW}\end{array}$ & $\begin{array}{c}Q_{n} \\
\mathrm{kVAr}\end{array}$ & $\begin{array}{c}P_{z} \\
\mathrm{~kW}\end{array}$ & $\begin{array}{c}Q_{z} \\
\mathrm{kVAr}\end{array}$ \\
\hline \hline Building & -4.16 & 0.00 & 0.00 & 0.00 & 0.00 & 0.00 \\
Load & 25.28 & 7.88 & -0.03 & -0.18 & -0.26 & 0.43 \\
Aggregat. & 29.45 & 7.88 & -0.03 & -0.18 & -0.26 & 0.43 \\
Dispatch. & 10.00 & 2.70 & -0.01 & -0.06 & -0.08 & 0.13 \\
Storage & 9.28 & 2.48 & -0.01 & -0.06 & -0.10 & 0.17 \\
Renew. & 10.00 & 2.70 & -0.01 & -0.06 & -0.08 & 0.13
\end{tabular}

Table 3. Positive, negative and zero sequence powers and peak currents in the whole building. (Scenario 1).

\section{Case of Study}

In this section, two different scenarios will be studied. In both of them the BMS was implemented in the Bus 2 of a four busses radial feeder (see Fig. 2) . Bus 0 is considered to be the coupling point of the SDN with the main grid (a slack bus). In busses 1 and 3, two conventional buildings were placed (B1 and B3). The building at bus 1 (B1) is consuming $11 \mathrm{~kW}, 13 \mathrm{~kW}$ and $15 \mathrm{~kW}$ in phases $a, b$ and $c$ respectively. The building in bus 3 (B3) is consuming $16 \mathrm{~kW}$, $6 \mathrm{~kW}$ and $14 \mathrm{~kW}$ in phases $a, b$ and $c$ respectively. All loads have a 0.95 power factor.

The Building placed at bus $2\left(\mathrm{nZEB}_{2}\right)$, is a nZEB with a Dispatchable generator, a PV (Renewable) generator and a Lithium-Ion based storage system. The building inner load is $5 \mathrm{~kW}, 7 \mathrm{~kW}$ and $13 \mathrm{~kW}$ in phases $a, b$ and $c$ respectively wit a 0.95 power factor (see Table 2). The maximum power that all generators can deliver is $10 \mathrm{~kW}$. The maximum peak current per phase at each converter is 35A. The line resistance and inductance are respectively $0.2 \Omega / \mathrm{km}$ and $20 \mathrm{mH} / \mathrm{km}$. No mutual coupling was considered.

In the first scenario, the PV generator is providing the maximum power and the battery is not empty so it can provide the maximum power too. The Building droop slope is set to $k=5 A / V$. This value is considered as an input provided by the SDN central controller and its calculation is beyond the scope of this paper. The higher the droop 


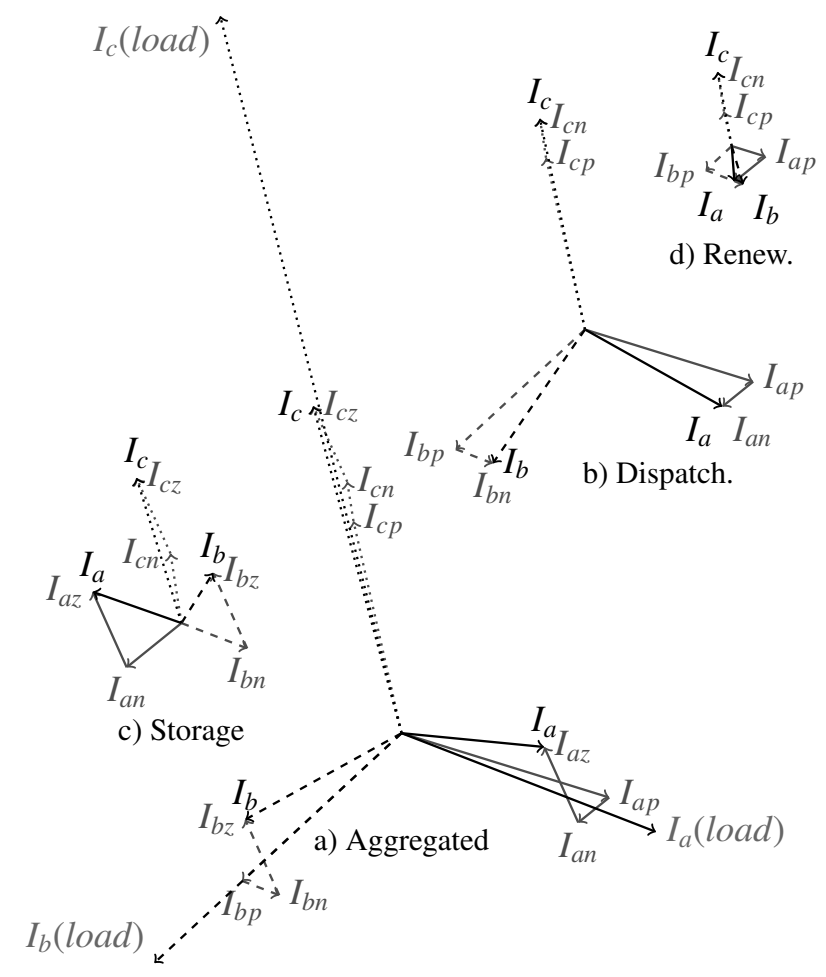

Figure 4. Phasor diagram of the optimised current injection in all converters and also the aggregated phasor diagram considering all converters (Scenario 2).

slope the higher the voltage support request from SDN central controller to the building. All converters have a four-leg configuration. In this case, the BMS is able to compensate all the internal consumption and also provides support to the grid injecting active power in positive sequence. In Table 1 the sequence voltages at nZEB point of coupling with the network are represented. Table 2 contains all powers and currents in $a b c$ reference. The net power consumed by the building in this case is negative because the building is supporting the network with a total injection of $4.2 \mathrm{~kW}$. The self compensation is achieved, so the reactive power consumed by the building loads is provided from inside. The current injected in the grid due to the P-V droop is in phase with the voltage, so no reactive power is exchanged between the building and the network. As it can be observed, in each phase, the power provided by the aggregation of the converters is higher than the power demanded by the loads.

It is important to mention that even when the content of negative and zero sequence currents in the load is important (see Table 3), the converters can compensate such sequences with a minimum amount of negative and zero sequence power, because the negative and zero voltages are very low and most of the consumed power is positive sequence power. As it can be also observed in Table 3, because the negative and zero compensation is fully achieved the net building zero and negative active power is null. In Fig. 3, a phasor plot represents the current injection of each converter and the aggregated currents injected by the three of them.

It has to be remarked that the maximum current per phase was achieved in phase $c$ of the storage device converter, so it can not provide its maximum power and it only injects $9.2 \mathrm{~kW}$. The Dispatchable generator injects the maximum power $10 \mathrm{~kW}$ and the renewable generator injects the available power $(10 \mathrm{~kW})$ in this case.

In the second scenario, the battery is empty, so it cannot provide active power. The loads in all buildings are the same for the first scenario. The available renewable (PV) power is $2 \mathrm{~kW}$ and the converter of the dispatchable generator and the renewable generator (PV) are configured as three-legs converters, so they cannot inject zero sequence currents. In this case of study, the full self-compensation is not achieved, so the fourth optimisation stage is not launched. The best that the BMS can do with the available resources is represented in Table 4 . A phasor diagram of this case is represented in Fig. 4. Most of the renewable power is injected in phase $c$. The battery is empty so it does not inject net active power (the positive sequence current in the battery is zero). However, the battery converter absorbs active 


\begin{tabular}{c|cc|cc|cc|cc} 
& $P_{a}$ & $Q_{a}$ & $P_{b}$ & $Q_{b}$ & $P_{c}$ & $Q_{c}$ & \multicolumn{2}{|c}{ Total } \\
& $\mathrm{kW}$ & $\mathrm{kVAr}$ & $\mathrm{kW}$ & $\mathrm{kVAr}$ & $\mathrm{kW}$ & $\mathrm{kVAr}$ & $\mathrm{kW}$ & $\mathrm{kVAr}$ \\
\hline \hline Building & 3.83 & 0.09 & 4.03 & 2.62 & 5.13 & 1.57 & 13.0 & 4.3 \\
Load & 5.00 & 1.64 & 7.00 & 2.30 & 13.00 & 4.20 & 25.0 & 8.1 \\
Aggregat. & 1.17 & 1.55 & 2.96 & -0.32 & 7.87 & 2.63 & 12.0 & 3.9 \\
Dispatch. & 2.73 & 1.35 & 3.52 & 0.35 & 3.75 & 1.37 & 10.0 & 3.1 \\
Storage & -1.64 & -0.48 & -1.17 & -0.05 & 2.82 & 0.76 & 0.0 & 0.2 \\
Renew. & 0.08 & 0.68 & 0.61 & -0.62 & 1.30 & 0.51 & 2.0 & 0.6 \\
\hline & \multicolumn{2}{|c|}{$I_{a}\left(\mathrm{~A}^{\circ}{ }^{\circ}\right)$} & \multicolumn{2}{|c|}{$I_{b}\left(\mathrm{~A}^{\circ}{ }^{\circ}\right)$} & \multicolumn{2}{c}{$I_{c}\left(\mathrm{~A},{ }^{\circ}\right)$} & \multicolumn{2}{|c}{$I_{n}\left(\mathrm{~A},{ }^{\circ}\right)$} \\
& Mod. & Ang. & Mod. & Ang. & Mod. & Ang. & Mod. & Ang. \\
\hline \hline Building & 3.83 & 0.09 & 4.03 & 2.62 & 5.13 & 1.57 & 13.0 & 4.3 \\
Load & 5.00 & 1.64 & 7.00 & 2.30 & 13.00 & 4.20 & 25.0 & 8.1 \\
Aggregat. & 1.17 & 1.55 & 2.96 & -0.32 & 7.87 & 2.63 & 12.0 & 3.9 \\
Dispatch. & 2.73 & 1.35 & 3.52 & 0.35 & 3.75 & 1.37 & 10.0 & 3.1 \\
Storage & -1.64 & -0.48 & -1.17 & -0.05 & 2.82 & 0.76 & 0.0 & 0.2 \\
Renew. & 0.08 & 0.68 & 0.61 & -0.62 & 1.30 & 0.51 & 2.0 & 0.6
\end{tabular}

Table 4. Powers and peak currents in $a b c$ reference in the whole system. The building net power is positive when it is demanded from the grid. The converter aggregated power is positive when it is delivered to the building (Scenario 2).

power from phases $a$ and $b$ and injects it to phase $c$. The zero sequence compensation is not fully achieved, only the battery converter injects zero sequence current. Even when the full compensation is not possible, the BMS makes the building more balanced reducing the harmful impact of the unbalanced load over the network.

It must be pointed out that with this technique, and even when the used droop is a P-V droop, the reactive power support is not neglected. In fact, the building tries to compensate its own reactive power consumption providing in this way an effective reactive power support. With this approach, the BMS does not try to compensate the reactive power consumed by the low-voltage lines which in fact is very small since the $\mathrm{X} / \mathrm{R}$ ration is extremely low $(0.32$ in our particular case of study). It does not try to provide either reactive power for other buildings just of itself. For instance, in the scenario 1 (see Table 2), the building load is consuming 8.1kVAr, while the net reactive power exchanged between the building and the grid is null, this is the ideal case in which the full compensation is achieved and all building reactive power consumption is produced inside the building. In a more critical case (scenario 2), where the full compensation cannot be achieved the load of the building is consuming $8.1 \mathrm{kVAr}$ but the net reactive power demanded from the grid is $4.3 \mathrm{kVAr}$ (see Table 4), so again, the building is providing some kind of reactive power support.

\section{Validation Setup}

The validation was carried out using the two AC low voltage feeders installed at the LEMUR Microgrid in the University of Oviedo. This microgrid is a highly configurable and flexible for testing different types of AC, DC and also Hybrid distribution topologies. It has two 400V AC four-wire feeders used in this case for validating the proposed approach. In [6], the whole hybrid system, the hardware, software, power flow equations, data bases and communication protocols are described. In Fig 5, the Lab setup and a snapshot of the SCADA system user interface developed specifically for this application can be observed.

For emulating the building loads, the authors obtained the input data from the smart meters installed by a local Distributor Network Operator (DNO) in 76 homes during one month. The input dataset includes the active and reactive powers recorded at each hour. In order to map the real data to the proposed structure, the input data is randomly grouped into the 2 internal nodes of each feeder representing the buildings. In [22] all details about the building load emulator installed in the Lab Setup can be found. Each feeder has two lines and each line is assumed to be $500 \mathrm{~m}$ long, with a line reactance to resistance ratio, $\mathrm{X} / \mathrm{R}=0.32$, and neglecting the cross-coupling between phases. The BMS system runs in a Raspberry PI B+ model installed at building level. All data from the buildings are sent to the central controller using Ethernet protocol.

A test with 100 cases has been conducted in order to validate the theoretical algorithms in the real Laboratory structure. Some of these tests can be analysed in [23]. In all cases, the errors when comparing theoretical and experimental cases are lower than $2 \mathrm{~V}$ (in Voltages), and 1A (In currents). In percentage, the error is lower than $0.9 \%$ 


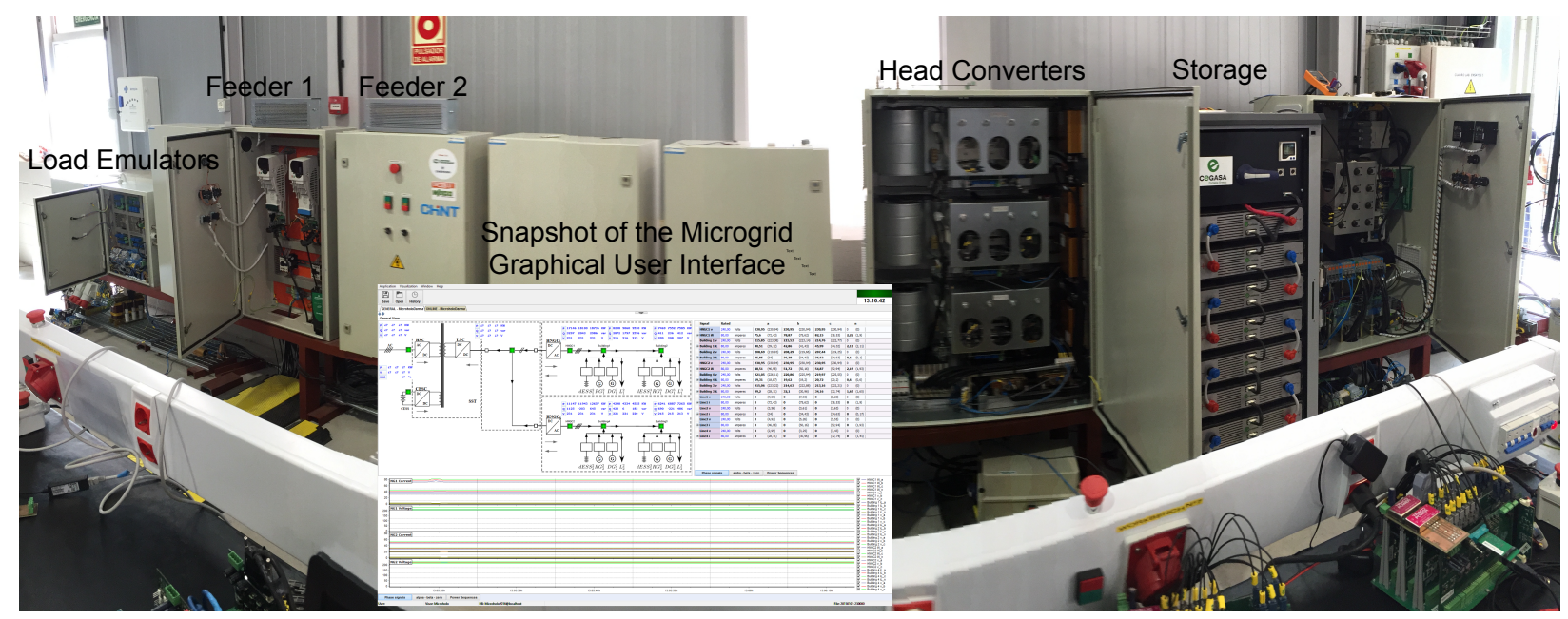

Figure 5. Laboratory setup for validation purposes implemented at LEMUR Microgrid (University of Oviedo) with a snapshot of the graphical user interface for real time microgrid monitoring.

in phase-to-neutral voltages and lower than $1 \%$ in currents. These errors do not mean that the theoretical model is not good or accurate enough, since the accuracy of the measurement process must be considered too. In our case, voltages and currents are acquired at each node as shown in [6]. Hall effect voltage and current sensors are provided with an accuracy of $0.1 \%$ over the entire input signal range. Signal adaptation for the interface with the A/D converters is implemented using analog electronics. The adaptation process includes a 2 nd order low-pass filter using a Butterworth Sallen-Key topology. $1 \%$ precision components are used for all the discrete elements in the chain. The $\mathrm{A} / \mathrm{D}$ converter is a 12 bit with an input range of $0.3 \mathrm{~V}$. The maximum voltage value accepted by the sensors is $1000 \mathrm{~V}$, whereas the current sensors are 200A. With those data, the input/output resolution is $7.5 \mathrm{mV} / \mathrm{A}$ and $1.5 \mathrm{mV} / \mathrm{V}$ for the current and voltage sensors respectively. Considering the A/D converter resolution is $0.7 \mathrm{mV} / \mathrm{bit}$, the final resolution for the current and voltage sensors is $0.1 \mathrm{~A} / \mathrm{bit}$ and $0.5 \mathrm{~V} / \mathrm{bit}$. Assuming some extra noise, is safe to say the resolution for the current could easily be in the range of $1 \mathrm{~A}$ and for the voltage in the range of $2 \mathrm{~V}$.

\section{Conclusions}

In this paper, the nZEBs with embedded converter interfaced generators have been used as a tool for reducing the network unbalances, and provide ancillary services like grid voltage support.

A cascaded optimisation coordination procedure among all generators and energy storage systems of the building has been implemented and tested. This cascade optimisation is implemented in the Building Management System (BMS), and it tries to compensate first the self consumed negative current, then the zero and then the positive. Finally, if the self compensation is achieved, the system tries to support the network by injecting/absorbing active power according to a P-V droop controller. In the paper it was also introduced an overall procedure for coordinating all BMS's with a Smart Distribution Network (SDN) central controller. In further works a joint model of the whole network with the central controller interacting with several nZEB will be developed. In order to implement this method in a real distribution feeder, all buildings must be monitored since active and reactive power measurements in all buildings are required for making the estimation of the impact of a given building into the network. In addition, for achieving better results in terms of voltage balance and network support, all buildings in the feeder should have this system implemented.

\section{References}

[1] A. Marszal, P. Heiselberg, J. Bourrelle, E. Musall, K. Voss, I. Sartori, A. Napolitano, Zero energy building: A review of definitions and calculation methodologies, Energy and Buildings 43 (4) (2011) 971 - 979. 
[2] F. Shahnia, P. Wolfs, A. Ghosh, Voltage unbalance reduction in low voltage feeders by dynamic switching of residential customers among three phases, Smart Grid, IEEE Transactions on 5 (3) (2014) 1318,1327.

\section{on Industrial Informatics 12 (4) (2016) 1426-1437.}

[22] D. Novak, M. Beraki, G. Villa, P. Garcia, Low-cost programmable three phase load for microgrids labs, in: Environment and Electrical

Engineering (EEEIC), 2015 IEEE 15th International Conference on, 2015, pp. 599-604.
[23] P. Arboleya, C. Gonzalez-Moran, P. Garcia, J. Garcia, B. Mohamed, Hierarchical coordination of a hybrid ac/de smartgrid with

[3] Z. Bao, Q. Zhou, Z. Yang, Q. Yang, L. Xu, T. Wu, A multi time-scale and multi energy type coordinated microgrid scheduling solution;part i: Model and methodology, Power Systems, IEEE Transactions on 30 (5) (2015) 2257,2266.

[4] Z. Bao, Q. Zhou, Z. Yang, Q. Yang, L. Xu, T. Wu, A multi time-scale and multi energy type coordinated microgrid scheduling solution; part ii: Optimization algorithm and case studies, Power Systems, IEEE Transactions on 30 (5) (2015) 2267,2277.

[5] Y. Cao, Y. Tan, C. Li, C. Rehtanz, Chance constrained optimization-based unbalanced optimal power flow for radial distribution networks, Power Delivery, IEEE Transactions on 28 (3) (2013) 1855,1864.

[6] P. Garcia, P. Arboleya, B. Mohamed, A. Vega, M. Vega, Implementation of a hybrid distributed/centralized real-time monitoring system for a dc/ac microgrid with energy storage capabilities, IEEE Transactions on Industrial Informatics PP (99) (2016) 1-1.

[7] Y.-R. Mohamed, E. El-Saadany, Adaptive decentralized droop controller to preserve power sharing stability of paralleled inverters in distributed generation microgrids, Power Electronics, IEEE Transactions on 23 (6) (2008) 2806,2816.

[8] M. Golsorkhi Esfahani, D. Lu, A control method for inverter based islanded microgrids based on v i droop characteristics, Power Delivery, IEEE Transactions on 30 (3) (2015) 1196,1204.

[9] M. Golsorkhi Esfahani, D. Lu, A decentralized control method for islanded microgrids under unbalanced conditions, Power Delivery, IEEE Transactions on PP (99).

[10] T. L. Vandoorn, J. D. M. D. Kooning, B. Meersman, J. M. Guerrero, L. Vandevelde, Automatic power-sharing modification of p / v droop controllers in low-voltage resistive microgrids, IEEE Transactions on Power Delivery 27 (4) (2012) 2318-2325.

[11] T. Vandoorn, J. V. de Vyver, B. Meersman, B. Zwaenepoel, L. Vandevelde, Phase unbalance mitigation by three-phase damping voltage-based droop controllers in microgrids, Electric Power Systems Research 127 (2015) 230 - 239.

[12] N. M. M, N. Sasidharan, J. G. Singh, A droop control incorporated dc equivalent power flow method for distribution and low voltage systems, Electric Power Systems Research 134 (2016) 56- 65.

[13] C. K. Sao, P. W. Lehn, Control and power management of converter fed microgrids, IEEE Transactions on Power Systems 23 (3) (2008) 1088-1098.

[14] X. Zhou, F. Tang, P. Loh, X. Jin, W. Cao, Four leg converters with improved common current sharing and selective voltage quality enhancement for islanded microgrids, Power Delivery, IEEE Transactions on PP (99).

[15] P. Arboleya, C. Gonzalez Moran, M. Coto, A hybrid central distributed control applied to microgrids with droop characteristic based generators, in: Power Electronics and Motion Control Conference (EPE/PEMC), 2012 15th International, 2012.

[16] H. Karami, M. Sanjari, S. Hosseinian, G. Gharehpetian, An optimal dispatch algorithm for managing residential distributed energy resources, Smart Grid, IEEE Transactions on 5 (5) (2014) 2360,2367.

[17] M. Bozchalui, S. Hashmi, H. Hassen, C. Canizares, K. Bhattacharya, Optimal operation of residential energy hubs in smart grids, Smart Grid, IEEE Transactions on 3 (4) (2012) 1755,1766.

[18] P. Arboleya, E. X. Dominguez, F. M. Lorenzo, Unified generating and storing capacity reliability evaluation in nearly zero energy buildings, in: Power Energy Society General Meeting, 2015 IEEE, 2015.

[19] D. Stimoniaris, T. Kollatou, D. Tsiamitros, M. Zehir, A. Batman, M. Bagriyanik, A. Ozdemir, E. Dialynas, Demand-side management by integrating bus communication technologies into smart grids, Electric Power Systems Research 136 (2016) 251 - 261.

[20] A. Alimardani, F. Therrien, D. Atanackovic, J. Jatskevich, E. Vaahedi, Distribution system state estimation based on nonsynchronized smart meters, IEEE Transactions on Smart Grid 6 (6) (2015) 2919-2928. central/distributed energy storage, in: 2016 IEEE Energy Conference and Exposition (ECCE), 2016, pp. 1-6. 